\title{
Disorders of sex development -A complex puzzle for the doctor and the patient-A rare case report
}

\author{
Kusum Jashnani ${ }^{1}$, Vivek Parameshwar, ${ }^{2,}$, Sneha D. Sirodkar ${ }^{3}$ \\ ${ }^{1,3}$ Professor \& HOD, ${ }^{2}$ Junior Resident, ${ }^{1,2}$ Dept. of Pathology, ${ }^{3}$ Dept. of Obstetrics and Gynaecology, B.Y.L. Nair Charitable \\ Hospital, Mumbai, Maharashtra, India
}

*Corresponding Author:

Email: choodamani.parameshwar@gmail.com

\begin{abstract}
Disorders of Sex Development (DSD) are referred to a group of disorders in which at least two out of gonadal sex, genetic sex, phenotypic sex and genital tract sex show discordance between them. Among the three main categories of DSD, 46XY DSD is a rarer type which we encountered in our case. A 14 year old female presented with abdominal pain and lump since 10 days. On clinical examination she had external sexual characters of a female. Karyotyping revealed a 46XY type. Intra-operatively, there was a normal uterus with a tumor mass in the location of the right gonad which was removed, along with a biopsy taken from the contralateral gonad, both intra-abdominal in location. Histopathologic examination of the tumor mass revealed a malignant mixed germ cell tumour (GCT) comprising of dysgerminoma and yolk sac tumour and the contralateral gonad was found to be testis, with evidence of intratubular germ cell neoplasm unclassified (IGCNU). Patient underwent 4 cycles of chemotherapy after the histopathologic diagnosis but was later lost to follow-up. The relative ambiguity of these disorders with their propensity to develop into type II GCTs makes these cases unique, perplexing and of utmost importance.
\end{abstract}

Keywords: 46XY DSD, Mixed GCT, IGCNU.

\section{Introduction}

The term DSD refers to disorders in which there is disagreement between chromosomal, gonadal and phenotypic sex due to defects in the normal process of sexual development. ${ }^{1}$ To reduce uncertainties on description, a revised classification system at the Chicago Consensus Meeting in 2005 was proposed, primarily based on the karyotype resulting in three categories: Sex chromosomal DSD, 46XY DSD and 46XX DSD. ${ }^{2,3}$ DSDs have been identified as one of the main risk factors for the development of type II GCT. ${ }^{4}$ Considering all these factors we are presenting a rare case of $46 \mathrm{XY}$ DSD with malignant mixed GCT and IGCNU in intraabdominal gonads which we came across at our institute.

\section{Case Report}

A 14 year old female presented with complaints of pain in abdomen and lump since 10 days. Further history revealed that she had not attained menarche and had noticed enlargement of breasts since 6 months. On clinical examination it was found that vaginal opening was present along with clitoral enlargement but sparse axillary and pubic hair. There was no family history of previously affected relatives. Further evaluation showed serum testosterone and 17 hydroxy progesterone levels to be within normal limits. USG was suggestive of a tumour mass on the right side and a gonad on the left side. CT scan revealed a large heterogeneous mass arising from the pelvis suggesting an ovarian neoplasm on the right side. Karyotyping by $\mathrm{G}$ banding peripheral blood was done which revealed a 46XY karyotype. She was operated upon and a normal uterus was found with a tumor mass on the right side and a small streak gonad on the left side. The mass was removed and sent for histopathological examination while a biopsy was taken from the left gonad.

\section{Specimen}

Gross Examination - Large tumour mass measuring $8 \times 5 \times 4.5 \mathrm{~cm}$ with $4 \mathrm{~cm}$ long fallopian tube on external surface along with fimbriae was seen. External surface of the tumor was grey white and nodular (Fig. 1a). Cut surface showed variegated appearance with one end showing a well circumscribed, demarcated area which was oval, brown in colour, homogenous and firm in consistency, with rest of the tumor mass being yellow-white in colour, soft, friable, predominantly solid with areas of haemorrhage and necrosis (Fig. 1b).

Histopathologic examination- The mass showed a malignant mixed germ cell neoplasm. One component was a dysgerminoma with characteristic large size tumour cells with clear cytoplasm and vesicular nuclei interspersed with lymphocytes (Fig. 2). The second component was yolk sac tumour with a characteristic reticular pattern of tumour cells and Schiller Duval bodies (Fig. 3). No normal ovarian or testicular stroma could be identified despite thorough sampling. Sections from the fallopian tube showed normal fallopian tube architecture.

A biopsy from the left gonad revealed seminiferous tubules showing maturation arrest and lined only by sertoli cells, with surrounding stroma showing leydig cells along with areas of micro calcifications within the seminiferous tubules. There was also evidence of occasional large atypical bizarre cells in the seminiferous tubules with enlarged hyperchromatic nucleus and scant cytoplasm suggestive of an IGCNU 
(Fig. 4) which was confirmed on immunohistochemistry showing PLAP and c-KIT positivity indicating their embryonal origin (Fig. 5) Based on these findings a final diagnosis of $46 \mathrm{XY}$ DSD with malignant mixed GCT of the right gonad with dysgenetic testis on the left showing IGCNU was made.

Follow up- After surgery, 4 out of the 6 cycles of chemotherapy for malignant GCT were administered. A gonadectomy for the left testis after completion of the chemotherapy followed by induction of oestrogen, followed by progesterone was planned since the patient as well as her family were satisfied with the same female gender as she had been raised. It was planned so as sex assignment is done only after careful medical, surgical and psychological assessment in full consultation between the medical team, the patient and his or her family. However the patient was lost to follow-up.

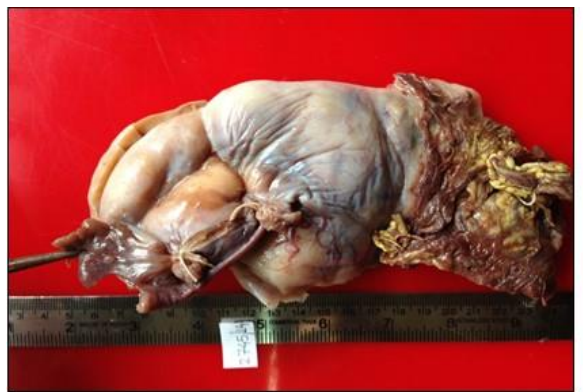

(a)

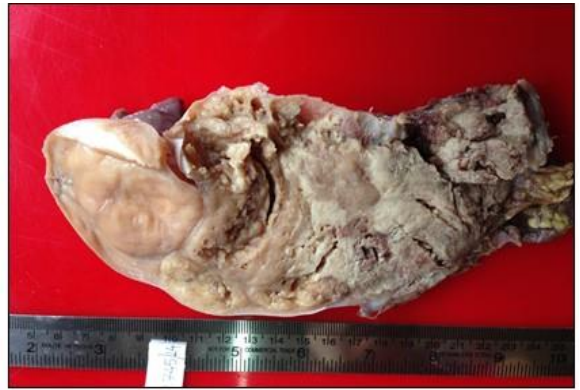

(b)

Fig. 1: Tumour mass. Gross examination - External surface (1a): Fallopian tube identified, tumor being grey white, nodular. Cut surface; (1b): variegated with brown, firm, circumscribed, demarcated area on the left; rest being yellow-white, friable, solid with haemorrhage, necrosis

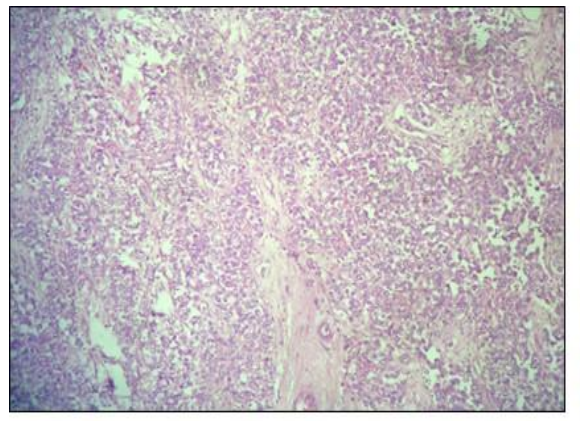

(a)

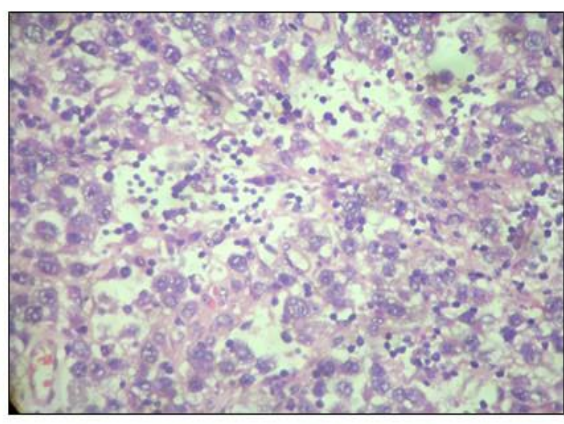

(b)

Fig. 2: Tumour mass. Microscopy H\&E stain - 40X; (2a) and 400X; (2b) magnification showing dysgerminoma with characteristic large size tumour cells with clear cytoplasm and vesicular nuclei interspersed with lymphocytes

(s)

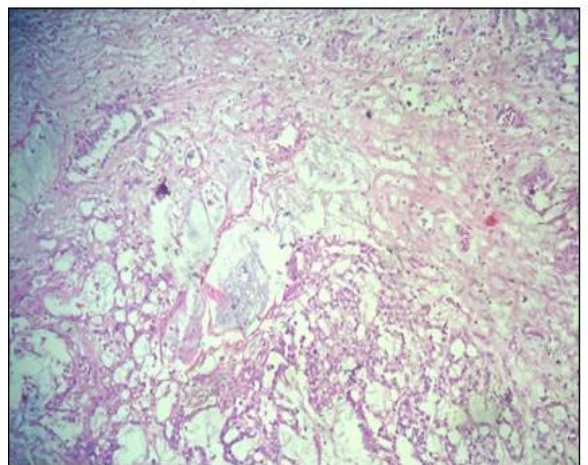

(p)

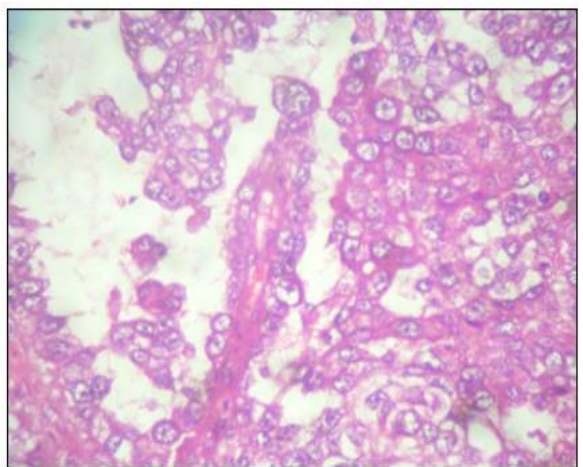

Fig. 3: Tumour mass. Microscopy- 40X; (3a) and 400X; (3b) magnification showing yolk sac tumour with a characteristic reticular pattern of tumour cells and Schiller Duval bodies 


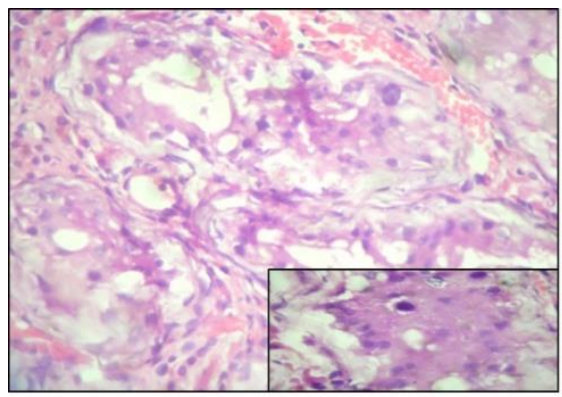

(a)

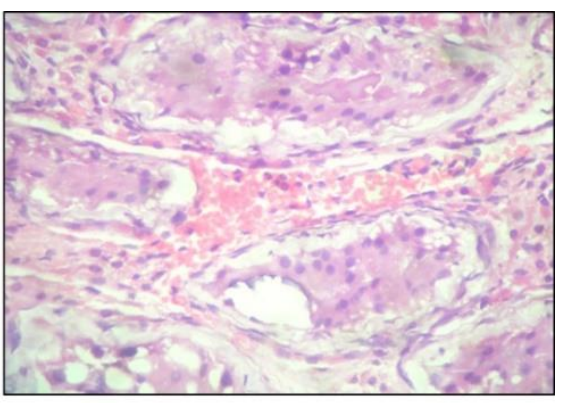

(b)

Fig. 4: Left gonad microscopy- 400X (4a) magnification showing seminiferous tubules with maturation arrest, lined by sertoli cells and evidence of occasional atypical bizarre cells having enlarged hyperchromatic nucleus, scant cytoplasm; indicating IGCNU; (4b) shows intratubular micro calcification

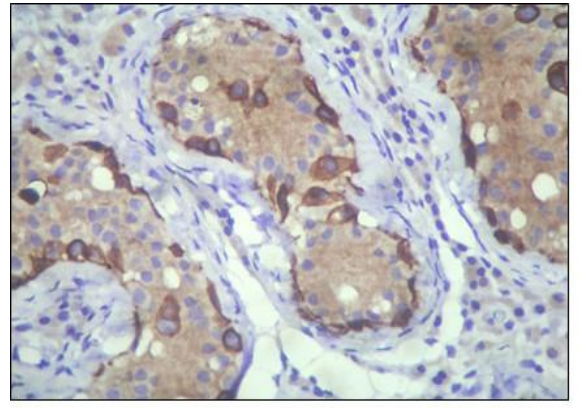

(a)

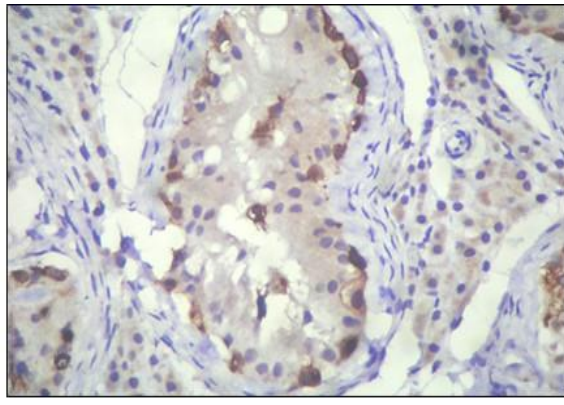

(b)

Fig. 5: Left gonad Immunohistochemistry-showing PLAP (5a) and c-KIT (5b) positivity confirming embryonal origin of IGCNU cells

\section{Discussion}

Individuals with disagreement between gonadal sex and external genitalia having a 46XY karyotype are classified as those with 46XY DSD. Our patient had a karyotype of 46XY with external genitalia of a female and a testis intra-abdominally leading to her classification in this category. Most of them have an autosomal recessive pattern of inheritance related to $\mathrm{X}$ chromosome. ${ }^{5,6}$ The incidence of DSD is estimated to be approximately 1 in 4500-5500 and those with $46 \mathrm{XY}$ DSD to be further lower at 1 in 20,000 births. ${ }^{7,8}$

Etiological factors include Leydig cell hypoplasia, enzyme disturbances of testosterone synthesis, deficiency of 5-alfa-reductase enzyme (DEF5 $\alpha$ ), gonadal dysgenesis (GD), testicular regression syndrome, androgen insensitivity syndrome (AIS) or ovotesticular DSD. The exact cause however in most of the cases cannot be pinpointed. ${ }^{9-11}$ As per Bianca Costa Mota et.al, maximum number of cases had no identifiable cause of DSD. Among the identifiable causes, most common was complete androgen insensitivity syndrome (CAIS) followed by partial androgen insensitivity, DEF5 $\alpha$, GD and ovotesticular DSD. ${ }^{1}$ Even in our case it was difficult to pinpoint an exact cause, but it could be androgen insensitivity, GD or ovotesticular DSD in all probability. Patients with 46XY DSD show a spectrum of patterns of external genitalia-from a male looking phallus to almost normal female genitalia with slight enlargement of clitoris with an intra-abdominal or inguinal testis. ${ }^{1}$ Most cases of DSD come to light in the new born period, when ambiguous genital development is noticed on the infant's first physical examination. Sometimes discordance between the prenatal karyotype, the prenatal ultrasound report and the genital appearance prompts an evaluation. Later presentations can occur in children, adolescents and adults. Examples of clinical findings associated with later presentation include progressive clitoromegaly, inguinal/labial masses in a phenotypic girl, etc. ${ }^{12}$ In our case, the patient had normal female genitalia with mild clitoral enlargement and intra-abdominal gonads. The presence of Y chromosome and probable cerebral masculinization, many a times characterises them as males. However, patients with CAIS and GD present a better adaptation to female sex which could be as in our case. ${ }^{6}$ The diagnosis of patients with 46XY DSD is mainly on the basis of clinical and laboratory evaluation. ${ }^{13}$ Patients with DSD have an increased risk of cancers of germ cell lineage mainly GCTs compared to the general population. ${ }^{12}$ Out of the 5 categories of GCTs, type II GCTs can be formed only in cases of retention of primordial germ cells (PGC) or gonocyte-like cells, delayed maturation being a risk factor as in DSDs. This can be due to both genetic as well as environmental factors in DSDs and can result in the formation of either gonadoblastoma (GB) or IGCNU of the testis, established by studies to be origin and precursor lesion 
of type II GCTs, the latter was seen in our case. ${ }^{4,15} \mathrm{~A}$ defined number of parameters are important in determining to what extent an individual is at risk. These include genomic constitution, i.e., the presence of Gonadoblastoma on the Y chromosome [GBY] region, and of the current best candidate gene TSPY; the expression of the embryonic germ cell markers OCT3/4 (POU5F1) and/or KITL (stem cell factor) beyond the age of 1 year, and the anatomical localization of the gonads. ${ }^{12}$ Type II GCTs are different than adult tumours in both pathobiology and clinical behaviour due to their origin. ${ }^{14}$ Histologically they are classified into seminomatous and nonseminomatous types. The seminomatous tumours are referred to as dysgerminoma of the ovary or dysgenetic gonad and immunohistochemically as well as morphologically mimic PGC. Within the group of non seminomatous tumours various variants can be identified like teratoma, yolk sac tumour and embryonal carcinoma. Tumours containing both the components are referred to as mixed GCTs as was in our case. ${ }^{4}$ Treatment comprises of gonadectomy with or without chemotherapy followed by hormonal therapy.

In summary, DSD patients with hyper virilisation are not at risk, while the risk of GCTs is highly variable in patients with hypo virilisation and gonadal dysgenesis. In general, it can be stated that the tumour risk decreases with a more accomplished maturation state of the gonad (ovary, testis, or even combined, i.e., ovotestis). ${ }^{4}$

\section{Conclusion}

A proper pathological classification can be the trigger for a full clinical description of a so-far undiagnosed, DSD patient, at high risk for tumor development.The early diagnosis and appropriate treatment of these disorders is of utmost importance not only from the medical but also from the social aspect. Proper decision making is very important considering the general young age at the time of clinical diagnosis and curability of the disease. Hence an active surveillance programme for the timely identification of such cases is the need of the hour.

\section{Conflict of Interest: None}

\section{References}

1. Mota BC. Clinical profile of 93 cases of $46 X Y$ disorders of sexual development in a referral centre. Int Braz J Urol 2015;41 (5):975-81.

2. Hughes IA, Houk C, Ahmed SF, Lee PA. Consensus statement on management of intersex disorders. Arch Dis Child 2006;91(7):554-63.

3. Hughes IA. Disorders of sex development: A new definition and Classification. Best Prac Res Clin Endocrinol Metab 2008;22(1):119-34.

4. Looijenga LH, et.al. Gonadal tumours and DSD. Best Pract Res Clin Endocrinol Metab 2010;24(2):291-310

5. Nussbaum RL, Mcinnes RR. Thompson \& Thompson. Genética Médica. Elsevier. 2008; ed. 7, pp. 111-4.

6. Intersex Society of North America. How common is intersex? [Internet] <http://www.isna.org/faq/ frequency>. Last accessed in: Sept 21, 2018.

7. Sax L. How common is intersex? A response to Anne Fausto-Sterling. J Sex Res 2002;39:174-78.

8. Nistal M, Paniagua R, Gonzalez-Peramato P, ReyesMugica M. Ovotesticular DSD (true hermaphroditism). Pediatr Dev Pathol 2015;18:345-52.

9. Abdullah MA. Disorders of sex development among Sudanese Children: 5-year experience of a paediatric endocrinology clinic. J Pediatric Endocrinol Metab 2012;25:1065-72.

10. Ng KL, Ahmed SF, Hughes IA. Pituitary-gonadal axis in male under masculinisation. Arch Dis Child 2000;82:54-8.

11. Chipashvili MK, Kristesashvili DI, Kopaliani N. Androgen Insensitivity syndrome in adolescents. Georgian Med News 2006;131:21-4.

12. Lee PA. Global Disorders of Sex Development Update since 2006: Perceptions, Approach and Care. Horm Res Paediatr. 2016; 85:158-180.

13. Damiani D, Setian N. Genitália Ambígua: Diagnóstico Diferencial e Conduta. Endocrinol Metab 2001;45:3747.

14. Oosterhuis J, Looijenga LH. Testicular germ cell tumours in a broader perspective. Nature Rev Cancer 2005;5(3):210-22.

15. Woodward PJ, Heidenreich A, Looijenga LH et al. Testicular germ cell tumors. In Eble JN, Sauter G, Epstein JI \& Sesterhann IA (eds.). World Health Organization classification of tumours pathology and genetics of the urinary system and male genital organs. Lyon: IARC Press. 2004, pg. 217-278.

How to cite the article: Jashnani K, Parameshwar V, Sirodkar S. D. Disorders of sex development -A complex puzzle for the doctor and the patient-A rare case report. Arch Cytol Histopathol Res 2018;3(4):227230. 